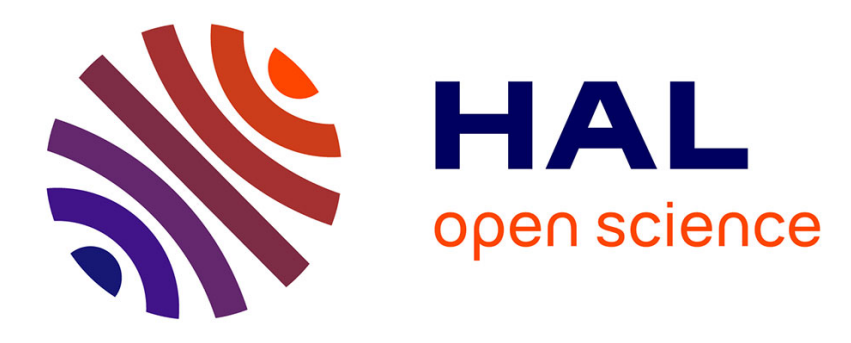

\title{
Low Speed Hybrid Generalized Predictive Control of a Gasoline-Propelled Car
}

Miguel Romero, Angel de Madrid, C Mañoso, V Milanés

\section{To cite this version:}

Miguel Romero, Angel de Madrid, C Mañoso, V Milanés. Low Speed Hybrid Generalized Predictive Control of a Gasoline-Propelled Car. ISA Transactions, 2015, 10.1016/j.isatra.2015.01.004 . hal01236978

\section{HAL Id: hal-01236978 https://hal.inria.fr/hal-01236978}

Submitted on 15 Dec 2015

HAL is a multi-disciplinary open access archive for the deposit and dissemination of scientific research documents, whether they are published or not. The documents may come from teaching and research institutions in France or abroad, or from public or private research centers.
L'archive ouverte pluridisciplinaire HAL, est destinée au dépôt et à la diffusion de documents scientifiques de niveau recherche, publiés ou non, émanant des établissements d'enseignement et de recherche français ou étrangers, des laboratoires publics ou privés. 


\title{
Low Speed Hybrid Generalized Predictive Control of a Gasoline-Propelled Car
}

\author{
M. Romero ${ }^{1 *}$, A. P. de Madrid ${ }^{1}$, C. Mañoso ${ }^{1}$, V. Milanés ${ }^{2}$, \\ ${ }^{1}$ Escuela Técnica Superior de Ingeniería Informática, UNED \\ Juan del Rosal, 16. 28040 - Madrid (Spain) \\ E-mail:mromero@scc.uned.es*; angel@scc.uned.es; carolina@scc.uned.es \\ ${ }^{2}$ RITS Team, INRIA Rocquencourt Domaine de Voluceau, \\ 78153 Le Chesnay, France \\ E-mail:vicente.milanes@inria.fr
}

*Corresponding Author

\begin{abstract}
Low-speed driving in traffic jams causes significant pollution and wasted time for commuters. Additionally, from the passengers' standpoint, this is an uncomfortable, stressful and tedious scene that is suitable to be automated. The highly nonlinear dynamics of car engines at low-speed turn its automation in a complex problem that still remains as unsolved. Considering the hybrid nature of the vehicle longitudinal control at low-speed, constantly switching between throttle and brake pedal actions, hybrid control is a good candidate to solve this problem.
\end{abstract}

This work presents the analytical formulation of a hybrid predictive controller for automated low-speed driving. It takes advantage of valuable characteristics supplied by predictive control strategies both for compensating un-modelled dynamics and for keeping passengers security and comfort analytically by means of the treatment of constraints. The proposed controller was implemented in a gas-propelled vehicle to experimentally validate the adopted solution. To this end, different scenarios were analysed varying road layouts and vehicle speeds within a private test track. The 
production vehicle is a commercial Citroën C3 Pluriel which has been modified to automatically act over its throttle and brake pedals.

Keywords: Hybrid control, predictive control, autonomous vehicle, longitudinal control. 


\section{Introduction}

Nowadays, the traffic jams represent a serious problem in large cities urban environments at rush hours wasting drivers' time and money daily. However, the worst consequence of this scene is the increase of human driver's mental fatigue due to the tedious task of control the vehicle speed in a very stressful condition.

Autonomous driving in traffic jams is an unresolved issue in the automotive sector [1]. The behaviour of production gasoline-propelled vehicle engines at low speeds exhibits highly nonlinear dynamics, making difficult its automation. Therefore, driving under these conditions constitutes one of the most important control challenges of this sector [2], where particular efforts have been put into developing automatic vehicle speed control with the aim of improving the safety of the car's occupants.

The first approaches to automatically handle vehicle speed were the cruise control (CC). These systems are based on controlling the accelerator pedal and maintain a set speed [3]; and the adaptive CC (ACC) where the action over the brake pedal to maintain a predefine gap with the preceding vehicle was introduced [2]. The widespread inclusion of these systems supposed an important reduction of car accidents with injuries [4]. Although some car makers include full-range speed control in their factory systems, they were mainly though for highway driving. Recent control systems are based on combining vehicle speed control with real-time information from the infrastructure to improve the traffic-flow, leading to intelligent CC (ICC) systems [5].

Specifically related to the longitudinal control at low-speed, a literature review shows several contributions for solving this problem using different control strategies. Among them, [6] compares PID and fuzzy control techniques for stop-and-go driving scenarios. In [7] a vision-based adaptive cruise control using fuzzy-logic is proposed. Finally, solutions based on fractional calculus have been proposed as in [8] where fractional PI controller was implemented; or [9] where a fractional predictive control was tested.

This paper deals with a hybrid predictive control strategy to solve this problem, combining the valuable characteristics of predictive control (fulfillment of constraints, robustness,...) with the hybrid nature of commercial vehicles (switching action over throttle and brake pedals). It brings about a suitable control strategy that compensates 
unmodeled dynamics and external disturbances. Moreover, the definition of process constraints allows to keep standing passengers security and comfort analytically.

On the one hand, Model Predictive Control (MPC) is an advanced process control methodology which has been widely adopted in industrial environments during the last 30 years. It has become an industrial standard due to its intrinsic ability to handle constrains for large scale multivariable plants $[10,11]$. It uses a dynamical model of the plant to predict and optimize the future behaviour of the process over a time interval known as the prediction horizon by means of a cost function minimization [12].

On the other hand, hybrid systems concept is usually described in the literature as the one that involves the interaction between continuous dynamics associated with differential or difference equations and logical/discrete components associated with finite state machines, Petri nets or other computation discrete models [13] (See Fig. 1). First works were carried out in the 90s using a two-stage control architecture. Low-level continuous physical processes were controlled by high-level finite-state logical components $[14,15]$. These systems attracted the attention of researchers both in academic and industrial environments because they provide an adequate framework for modelling real systems as: electrical circuits with diodes and transistors, temperature control by thermostat, mechanical transmission,... [16]. Furthermore, we can find theoretical works that consider this problem in terms of mixed logical dynamical systems (MLD) or piecewise affine systems (PWA) based on a space-state formulation[17, 18, 19]. Using these multiparametric programming techniques, the explicit resultant controller can be expressed as a piecewise affine function of a parameter vector which can be stored as a simple look-ahead table. In this context, there are two important tools for the computation of explicit controllers: the Multiparametic Toolbox [20, 21] and the Hybrid Toolbox [22]. However, the real-time code generated by both toolboxes is not compatible with our experimental vehicle, because it cannot be embedded in the car hardware directly. Therefore, in our case, we have used the $\mathrm{C}++$ programming language to code the proposed hybrid controller and to access to the hardware resources of our test vehicle properly.

There exist numerous examples of practical applications which use a hybrid strategy. We can mention, without intention to be exhaustive, some works such as [23] where it is presented a hybrid system approach to control power electronics systems, [24] where 
hybrid predictive control applications to chemical processes are described. Finally, in [25] an application for solving the dynamic multi-vehicle pick-up and delivery problem is discussed.

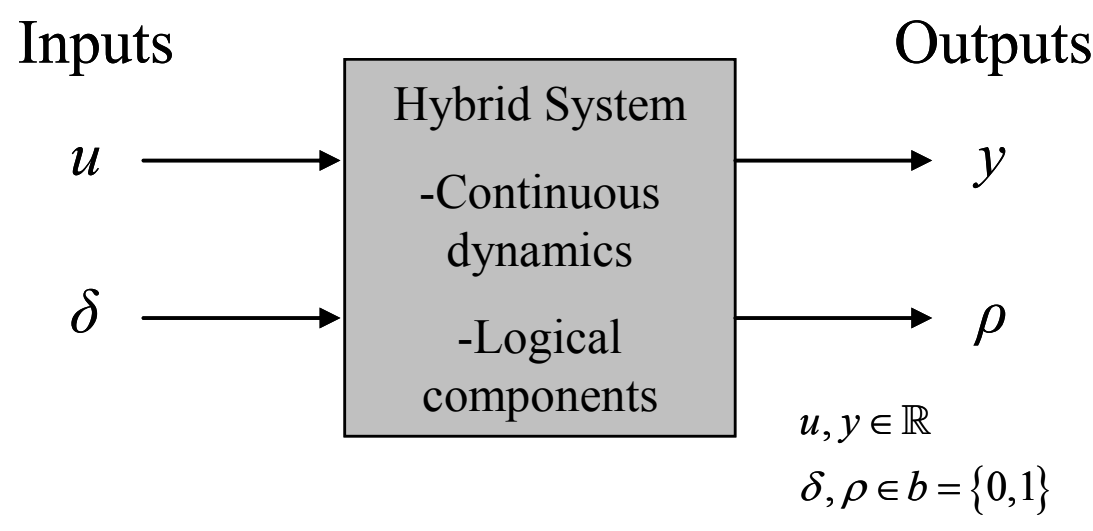

Fig. 1. Block diagram of hybrid system.

In brief, the main contributions of this papers are: (1) The development of a new hybrid controller based on a transfer function model in accordance with the chosen predictive control strategy to implement it. (2) The proposed controller has been trialled recreating the common low speed situation in traffic jams and fulfilling all control requirements in spite of uncertainties and circuit perturbations. (3) The comparison with other strategies has shown a better performance of our hybrid controller in terms of speed error.

Therefore, we work out the challenging topic that is unsolved by the automotive sector: managing autonomously a real gasoline car at low speed conditions. To do so, this paper is structured as follows: Section 2 summarizes the fundamentals of hybrid and MPC control and describes mathematically the formulation of the proposed control strategy. Section 3 includes the description of the experimental vehicle and the identification of its longitudinal dynamics. Section 4 presents the design and tuning of the hybrid controller for the experimental vehicle and Section 5 shows the results of the experimental trials following different itineraries and a comparison with other strategies. Finally, section 6 draws the main conclusions of this work.

\section{Hybrid controller formulation}

This section is divided into two parts. In the first part, the very well-known fundamentals of predictive control strategy are mentioned briefly, focusing on the so- 
called Generalized Predictive Control (GPC). In the second part, the formulation of the proposed hybrid control strategy is described mathematically, using GPC controllers to act over throttle and brake actions.

\subsection{Fundamentals of GPC}

GPC is one of the most representative MPCs due to its success both in industrial and in academic environments [26, 27]. It shares, together with the rest of predictive algorithms, the same concept $[10,11,12]$. At each present time $t$, GPC generates a set of future control signals $\Delta u(t+k \mid t)$ based on the prediction of the future process outputs $\hat{y}(t+k \mid t)$ by means of minimization of a cost function within a time window defined by $\left[N_{1}, N_{2}\right]$. Nevertheless, only the first term of the control signal vector is applied to the system input $\Delta u(t \mid t)$, the rest of them are neglected. In the following step, at time is equal to $t+1$, the previous algorithm is repeated to calculate the new corresponding system input $\Delta u(t+1 \mid t+1)$. Therefore, the prediction window has moved forward one step (receding horizon control). This control strategy is analogous to car's driver one as it is depicted in Fig. 2.

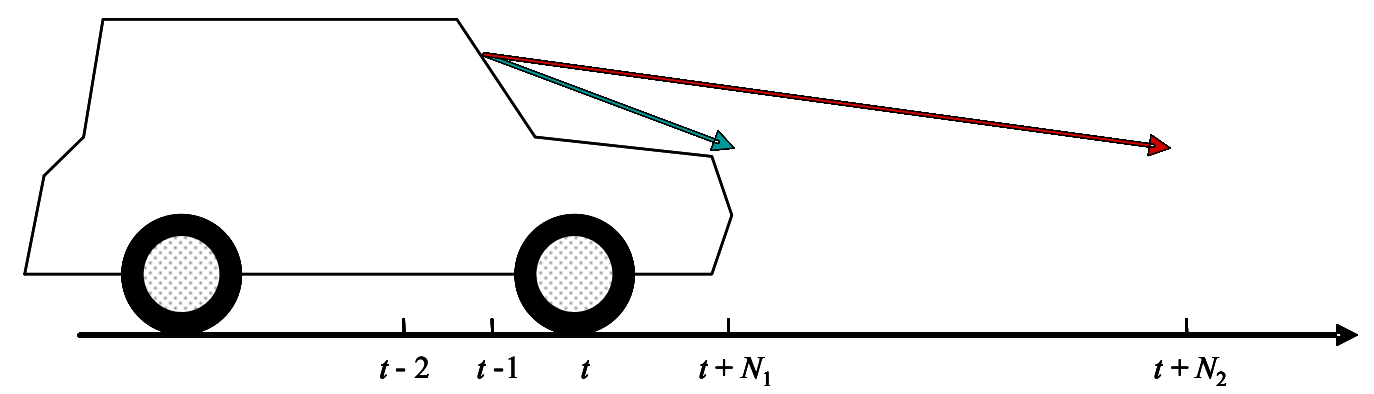

Fig. 2. Predictive control analogy.

The cost function that GPC minimizes subject to a set of constraints is

$$
J_{G P C}(\Delta u, t)=\sum_{k=N_{1}}^{N_{2}} \gamma_{i}(r(t+k)-y(t+k))^{2}+\sum_{k=1}^{N_{u}} \lambda_{i} \Delta u(t+k)^{2}
$$

where: $r$ is the reference, $u$ is the input, $y$ is the output, $N_{1}$ and $N_{2}$ are the costing horizons, $N_{u}$ is the control horizon, $\gamma$ is the future errors weighting sequence, $\lambda$ is the control weighting sequence and $\Delta$ is the increment operator.

Constraints are expressed as a set of linear inequalities 


$$
H \Delta u(k) \leq \bar{h}(k)
$$

where $H \in \mathbb{R}^{l x N_{u}}$ is a matrix, $\bar{h} \in \mathbb{R}^{l}$ is a vector, $l$ is the number of constraints and $\Delta u(k)$ is the vector that contains the future (predicted) control increments.

It is assumed that the control signal $u(t)$ remains constant from time instant $t+N_{u}$ $\left(1 \leq N_{u} \leq N_{2}\right)[26,27]$ and all expressions are referred to the present time $t$.

The GPC cost function has an equivalent matrix form

$$
J_{G P C}=e(k)^{\prime} \Gamma e(k)+\Delta u(k)^{\prime} \Lambda \Delta u(k)
$$

where $e(k)=r(k)-y(k)$ is the error vector and $\Gamma \equiv \operatorname{diag}\left(\gamma_{i}\right), \Lambda \equiv \operatorname{diag}\left(\lambda_{i}\right)$ are diagonal matrices that contain the future errors and control weighting sequences.

In order to predict the future output signal $\hat{y}(t)$, GPC makes use of a CARIMA (Controlled Auto-Regressive Integrated Moving-Average) model to describe the system dynamics

$$
A\left(z^{-1}\right) y(t)=B\left(z^{-1}\right) u(t)+\frac{T\left(z^{-1}\right)}{\Delta} \xi(t)
$$

where $B\left(z^{-1}\right)$ and $A\left(z^{-1}\right)$ are the numerator and denominator of the model transfer function, respectively, $T\left(z^{-1}\right)$ is a polynomial filter that improves the system robustness rejecting disturbance and noise $[28,29]$, and $\xi(t)$ represents uncorrelated zero-mean white noise.

The future output signal (5) has two parts. $y_{C}$ (forced response) is the future output part that depends on the future control action increment $\Delta u$ with $y_{C}=\mathrm{G} \cdot \Delta u$, and $y_{F}$ (free response) is the future output part that does not depends on the future control action increment $\Delta u ; G \in \mathbb{R}^{\left(N_{2}-N_{1}+1\right) x N_{u}}$ is a matrix to consist of the step response coefficients of the model.

$$
\hat{y}=y_{C}+y_{F}
$$

From (3) and (4) the cost function can be expressed as 


$$
J_{G P C}=\Delta u(k)^{\prime}\left(G^{\prime} \Gamma G+\Lambda\right) \Delta u(k)-2 E_{0}(k)^{\prime} \Gamma G \Delta u(k)+E_{0}(k)^{\prime} \Gamma E_{0}(k)
$$

with $E_{0}(k)=r(k)-y_{F}(k)$.

The free response, $y_{F}$, can be calculated by [12]

$$
y_{F}(k)=\Phi u^{f}(k)+F y^{f}(k)
$$

where "f" means a quantity filtered by $1 / T\left(z^{-1}\right)$ and $\Phi, F$ are two polynomials obtained from the resolution of two Diophantine equations (8) and (9). See [26, 27] for more details.

$$
\begin{gathered}
T=E_{j} A \Delta+z^{-j} F_{j} \\
E_{j} B=G_{j} T+z^{-j} \Phi_{j}
\end{gathered}
$$

with $j$ equals to the prediction interval.

The optimal control sequence is obtained by minimization of (3) subject to set of constraints (2).

$$
\Delta u^{*}(k)=\arg \min _{\Delta u} J_{G P C}
$$

Replacing expression (6) in equation (10) yields

$$
\Delta u^{*}(k)=\arg \min _{\Delta u}\left\{\frac{1}{2} \Delta u(k)^{\prime}\left(G^{\prime} \Gamma G+\Lambda\right) \Delta u(k)-E_{0}(k)^{\prime} \Gamma G \Delta u(k)\right\}
$$

Thus, the expression (11) together with the set of constraints (2) is agreed with the classical formulation of a quadratic programming problem (12) which can be solved numerically by computer.

$$
\Delta u^{*}(k)=\arg \min _{\Delta u}\left\{\frac{1}{2} \Delta u(k)^{\prime} K \Delta u(k)+L^{\prime} \Delta u(k)\right\}
$$

with $\Delta u(k)=u(k)-u(k-1)$. 
Since the term $K=G^{\prime} \Gamma G+\Lambda$ is constant due to a Linear Time Invariant (LTI) model is used (4), it can be initially calculate off-line to reduce the calculation time. However, the term $L=-G^{\prime} \Gamma E_{0}(k)$ is not constant because it depends on future predicted errors $E_{0}(k)$ and has to be calculated in each sampling time $\Delta t$.

\subsection{Integrated throttle and brake control}

The basic control of a commercial gasoline-propelled vehicle involves mainly two tasks: the throttle control and the brake control which have quite different dynamics. Moreover, both actions must be mutually exclusive in order to keep standing passengers security and comfort. This is a practical situation where physical dynamics and logic rules interact to characterize the plant dynamical behaviour, leading to a hybrid system[17].

The proposed hybrid control system in this paper is focused on low-speed driving. Bearing this in mind, gearbox will remain in first gear and it will not be taken into account to model vehicle's behaviour.

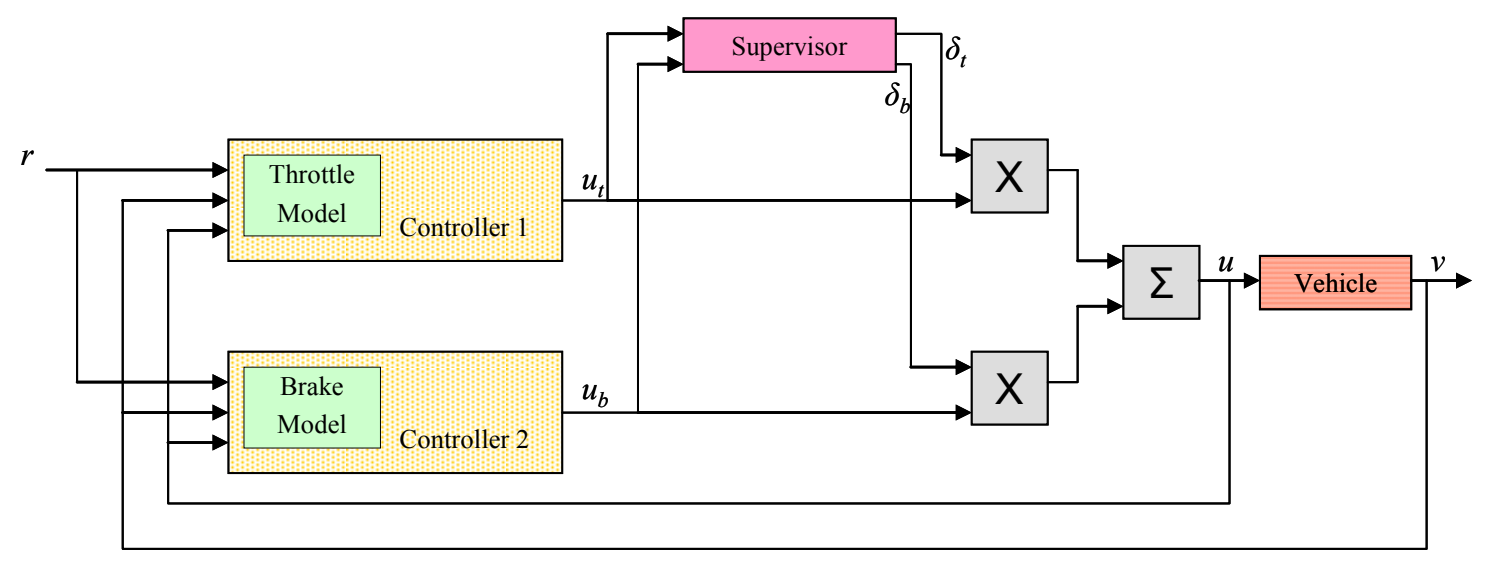

Fig. 3. Hybrid control system schema.

Fig. 3 illustrates the hybrid control system schema proposed in this work. It is constituted by two GPC controllers depicted by block "Controller 1" and block "Controller 2" which are tuned to fulfill closed-loop system specifications and an optimization criterion (follow speed reference $r$ ) subject to a set of possible constraints. To do so, each controller block has a CARIMA model that represents the physical dynamics to be controlled, throttle (13) and brake (14). 


$$
\begin{aligned}
& A_{t}\left(z^{-1}\right) y_{t}(t)=B_{t}\left(z^{-1}\right) u_{t}(t)+\frac{T_{t}\left(z^{-1}\right)}{\Delta} \xi(t) \\
& A_{b}\left(z^{-1}\right) y_{b}(t)=B_{b}\left(z^{-1}\right) u_{b}(t)+\frac{T_{b}\left(z^{-1}\right)}{\Delta} \xi(t)
\end{aligned}
$$

In order to obtain the optimum control values $u_{t}$ and $u_{b}$, the present and past values of vehicle velocity, $y$, and control variable, $u$, are needed to calculate the free response $y_{F}$ for each controller in each step, $k$.

$$
\begin{gathered}
y_{F_{t}}(k)=\Phi_{t} u_{t}^{f}(k)+F_{t} y_{t}^{f}(k) \\
y_{F_{b}}(k)=\Phi_{b} u_{b}{ }^{f}(k)+F_{b} y_{b}{ }^{f}(k)
\end{gathered}
$$

The future predicted errors for each controller can be calculated now using previous free responses.

$$
\begin{aligned}
& E_{0_{t}}(k)=r_{t}-y_{F_{t}}(k) \\
& E_{0_{b}}(k)=r_{b}-y_{F_{b}}(k)
\end{aligned}
$$

Using both predicted errors expressions, the no-constant term $L$ can be calculated for both controllers.

$$
\begin{gathered}
L_{t}=-G_{t}{ }^{\prime} \Gamma_{t} E_{0_{t}}(k) \\
L_{b}=-G_{b}{ }^{\prime} \Gamma_{b} E_{0_{b}}(k)
\end{gathered}
$$

Obviously, constant terms $K$ must be calculated once.

$$
\begin{gathered}
K_{t}=G_{t}{ }^{\prime} \Gamma_{t} G_{t}+\Lambda_{t} \\
K_{b}=G_{b}{ }^{\prime} \Gamma_{b} G_{b}+\Lambda_{b}
\end{gathered}
$$

In this point, two quadratic programming problems are formulated and ready to be numerically resolved. Each of them subject to own constraints. 


$$
\begin{aligned}
& \Delta u_{t}^{*}(k)=\arg \min _{\Delta u_{t}}\left\{\frac{1}{2} \Delta u_{t}(k)^{\prime} K_{t} \Delta u_{t}(k)+L_{t}{ }^{\prime} \Delta u_{t}(k)\right\} \\
& \text { subject to } H_{t} \Delta u_{t}(k) \leq \bar{h}_{t}(k) \\
& \Delta u_{b}^{*}(k)=\arg \min _{\Delta u_{b}}\left\{\frac{1}{2} \Delta u_{b}(k)^{\prime} K_{b} \Delta u_{b}(k)+L_{b}{ }^{\prime} \Delta u_{b}(k)\right\} \\
& \text { subject to } H_{b} \Delta u_{b}(k) \leq \bar{h}_{b}(k)
\end{aligned}
$$

The task of "Supervisor" block is to apply the switching rule that determines whether throttle or brake action is needed, by means of selecting the value of control variable $u$, depending on the sign of $u_{t}$ and $u_{b}$. In this work, we have followed the simple rule proposed in [30], because it works properly with the hybrid nature of commercial vehicles [31], can be programmed with ease and prevents the undesired chattering effect [6]. It can be summarized as follows:

- Throttle control region. If $\left(u_{t}>0\right.$ and $\left.u_{b}>0\right)$. The vehicle is in normal control state and the throttle control is active $u(t)=u_{t}(t)$.

- Brake control region. If $\left(u_{t}<0\right.$ and $\left.u_{b}<0\right)$. The vehicle is in emergence state and the brake control is active $u(t)=u_{b}(t)$.

- Switching control region. If $\left(u_{t}>0\right.$ and $\left.u_{b}<0\right)$ or $\left(u_{t}<0\right.$ and $\left.u_{b}>0\right)$. The vehicle is in undesired state and must change to throttle or brake region. In this case the zero control signal is chosen $u(t)=0$.

Thus, the control variable $u$ has the following expression:

$$
u(k)=\delta_{t} u_{t}(k)+\delta_{b} u_{b}(k)
$$

with $\delta_{t}, \delta_{b}=\{0,1\}$.

\subsubsection{Control constraints implementation}

It is well known that one of the most valuable characteristics of predictive control strategies is the possibility of using process constraints. Although, theoretically, it is possible to impose constraints on any variable related to system behaviour, only linearly dependent variables of control signal $\Delta u$ are used, due to quadratic programming 
algorithm restriction which allows only to satisfy constraints with general expression as (2).

Nevertheless, it is possible to regulate system variables in other ways. For instance, setting the controller parameters, specially $N_{2}, N_{u}$ and $\lambda$ to meet the requirements. This could be accomplished by trial and error or by optimization. (For applications of optimization methods to tune model predictive controllers see $[9,32]$.) However, in this work, all system requirements will be expressed as constraints both for the throttle control and for the brake control.

- Velocity constraint: the car speed $v(\mathrm{~km} / \mathrm{h})$ is the system output $y$. Let $v(k)$ be the vehicle speed at time instant $k$, whose values are within $v_{\text {Min }} \leq v(k) \leq v_{\text {Max }}$. Making use of prediction equation (5) and after a few algebraic manipulations, we can finally express velocity constraints in the general form (2):

$$
\left[\frac{-G}{G}\right] \cdot \overline{\Delta u}(k) \leq\left[\frac{-\left(v_{\text {Min }}-y_{F}\right)}{\left(v_{M a x}-y_{F}\right)}\right]
$$

- Acceleration constraint: Let $a(k)$ be the car acceleration at time instant $k$, whose values are within $a_{\text {Min }} \leq a(k) \leq a_{M a x}$. By definition,

$$
a(k)=\frac{\Delta v(k)}{\Delta t}=\frac{\Delta y(k)}{\Delta t}
$$

Thus,

$$
a_{\text {Min }} \Delta t \leq \Delta y(k) \leq a_{\text {Max }} \Delta t
$$

Hence, acceleration constraints in this system will be expressed as $\Delta y$ constraints in GPC formulation. Let us define

$$
\overline{\Delta y}(k) \equiv\left[\begin{array}{c}
\Delta y(k+1) \\
\Delta y(k+2) \\
\Delta y(k+3) \\
\vdots \\
\Delta y\left(k+N_{2}\right)
\end{array}\right]=\left[\begin{array}{c}
y(k+1)-y(k) \\
y(k+2)-y(k+1) \\
y(k+3)-y(k+2) \\
\vdots \\
y\left(k+N_{2}\right)-y\left(k+N_{2}-1\right)
\end{array}\right]
$$


Equation (29) can be obtained as a subtraction of two prediction equations (5) shifted one sampling time

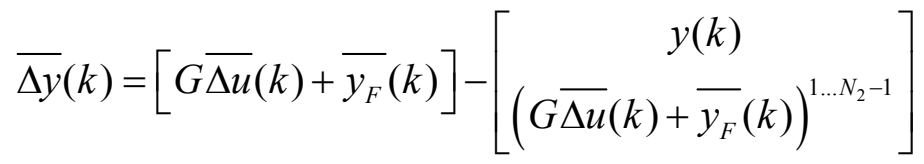

After a few algebraic manipulations, we obtain:

$$
\overline{\Delta y}(k)=\Delta G \cdot \overline{\Delta u}(k)+\overline{\Delta y_{F}}(k)
$$

with $\Delta G \equiv\left[\begin{array}{ccccc}g_{1} & 0 & 0 & \cdots & 0 \\ g_{2}-g_{1} & g_{1} & 0 & \cdots & 0 \\ g_{3}-g_{2} & g_{2}-g_{1} & g_{1} & \cdots & 0 \\ \vdots & \vdots & \vdots & \ddots & \vdots \\ g_{N_{2}}-g_{N_{2}-1} & g_{N_{2}-1}-g_{N_{2}-2} & g_{N_{2}-2}-g_{N_{2}-3} & \cdots & g_{1}\end{array}\right]$;

Using this result, we can finally express acceleration constraints (28) in the general form (2):

$$
\left[\frac{-\Delta G}{\Delta G}\right] \cdot \overline{\Delta u}(k) \leq\left[\frac{-\left(\Delta y_{M i n}-\Delta y_{F}\right)}{\Delta y_{M a x}-\Delta y_{F}}\right]
$$

- Input constraints: These constraints influence on the slew rate and on the amplitude of the manipulated variable $u$. In the following, it will be assumed that the manipulated variable is constrained to the interval $u_{\operatorname{Min}} \leq u(k) \leq u_{\text {Max }}$, having:

$$
\left[\begin{array}{cccc}
-1 & 0 & \cdots & 0 \\
-1 & -1 & \cdots & 0 \\
\vdots & \vdots & \ddots & \vdots \\
-1 & -1 & \cdots & -1 \\
\hline 1 & 0 & \cdots & 0 \\
1 & 1 & \cdots & 0 \\
\vdots & \vdots & \ddots & \vdots \\
1 & 1 & \cdots & 1
\end{array}\right] \cdot \overline{\Delta u}(k) \leq\left[\begin{array}{c}
-u_{\text {Min }}+u(k-1) \\
-u_{\text {Min }}+u(k-1) \\
\vdots \\
\frac{-u_{M i n}+u(k-1)}{u_{M a x}-u(k-1)} \\
u_{M a x}-u(k-1) \\
\vdots \\
u_{M a x}-u(k-1)
\end{array}\right]
$$

The control signal rate of change was also constrained within $\Delta u_{\operatorname{Min}} \leq \Delta u(k) \leq \Delta u_{\operatorname{Max}}$, obtaining: 


$$
\left[\begin{array}{cccc}
-1 & 0 & \cdots & 0 \\
-1 & -1 & \cdots & 0 \\
\vdots & \vdots & \ddots & \vdots \\
-1 & -1 & \cdots & -1 \\
\hline 1 & 0 & \cdots & 0 \\
1 & 1 & \cdots & 0 \\
\vdots & \vdots & \ddots & \vdots \\
1 & 1 & \cdots & 1
\end{array}\right] \cdot \overline{\Delta u}(k) \leq\left[\begin{array}{c}
-\Delta u_{M i n} \\
-\Delta u_{M i n} \\
\vdots \\
-\Delta u_{M i n} \\
\Delta u_{M a x} \\
\Delta u_{M a x} \\
\vdots \\
\Delta u_{M a x}
\end{array}\right]
$$

Positive control values shall be interpreted as an acceleration command and negative values as a brake command.

\section{Experimental vehicle}

In order to validate the proposed hybrid controller in a real environment, an instrumented real car with control devices was used. Specifically, it is a commercial convertible Citroën C3 Pluriel (Fig. 4) that is modified with automatic driving capabilities by hardware modifications to permit autonomous actions on the accelerator and brake pedals. These modifications allow the controller's outputs to act over vehicle's actuators. To do so, the vehicle is equipped with an on-board industrial PC situated at the boot which governs the necessary peripheral devices. It is in charge of sending the control signal generated to the actuators in each control cycle (200 ms.) 


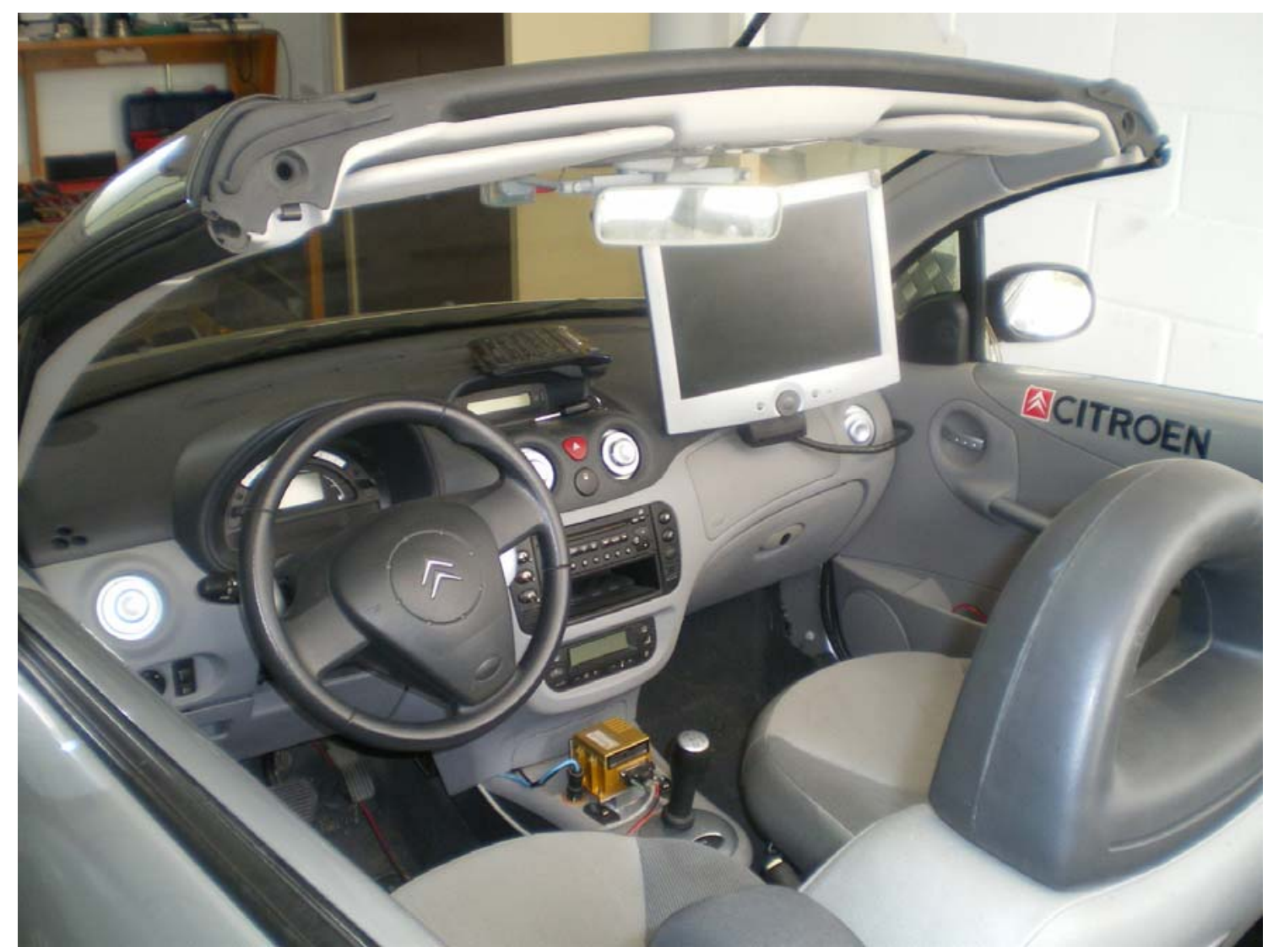

Fig. 4. Interior of experimental vehicle prototype.

The experimental car's throttle is handled by an analog signal that reproduces the pressure on the pedal generated by an analog card. The action over this pedal is transformed into two analogue values between 0 and $5 \mathrm{~V}$. A switch is installed to commute between automatic throttle control and original throttle circuit on the dashboard of vehicle.

On the other hand, the brake's automation has been carried out taking into account that its action is critical, that is, the brake-by-wire system must be capable of working in parallel with the original braking circuit. In this way, the autonomous system can be override by the driver to avoid potential risk situations when testing. So an electrohydraulic braking system is installed in parallel with the original one, permitting to coexist two systems independently. (Refer to $[33,34]$ for more details about vehicle automation.)

With respect to on-board sensor systems, a real-time kinematic-differential global positioning system (RTK-DGPS) together with an inertial unit (IMU) are used for vehicle positioning and location. Speed and acceleration of the car are provided by a 
differential hall effect sensor and a piezoelectric sensor, respectively. These values are acquired via controller area network bus (CAN) giving the necessary information to the hybrid control algorithm, that is running in real-time in the on-board industrial PC, generating the control actions to govern the actuators. This hybrid control algorithm has been coded in $\mathrm{C}++$ programming language following the object-oriented programming paradigm and using the appropriate device drivers to access to the CAN bus.

\subsection{Identification of the longitudinal dynamics}

The hybrid control strategy based on GPC controllers needs a CARIMA model of the plant to make the necessary predictions as we have seen previously. It is a fact that the gasoline-propelled vehicle dynamics are highly nonlinear, above all at very low speed (first gear) where a high engine brake force is acting. Moreover, the mechanism to generate braking efforts is the tire-road interaction. It is a very complex phenomenon which depends on poorly known factors [6]. So, finding an exact dynamical model for the vehicle is not a straightforward task. Nevertheless, in spite of these difficulties and others like road inclination, road surface state,...; an identification process has been carried out which provides two simplified models, one for each dynamics (throttle and brake), that represent adequately both events.

The manipulated variable in this identification process is Pedal, whose values are normalized within the range of $[-1,1]$ and determine which actuator has to be pressed. The brake pedal is completely pressed when Pedal $=-1$, and Pedal $=1$ means that the maximum action is applied to the accelerator pedal. The output variable is the speed of the vehicle in $\mathrm{km} / \mathrm{h}$.

The models for both dynamics (throttle and brake) of the vehicle obtained by means of an identification process with the MATLAB Identification Toolbox [35] have these discrete transfer functions: (35) and (36), respectively. The sampling time, $\Delta t$, is equal to the control cycle (200 ms.)

$$
\begin{aligned}
& \frac{B_{t}\left(z^{-1}\right)}{A_{t}\left(z^{-1}\right)}=\frac{5.1850 z^{-4}}{1-0.7344 z^{-1}-0.2075 z^{-2}} \\
& \frac{B_{b}\left(z^{-1}\right)}{A_{b}\left(z^{-1}\right)}=\frac{5.4230 z^{-4}}{1-1.5180 z^{-1}+0.5637 z^{-2}}
\end{aligned}
$$


In spite of environment and circuit perturbations, both models capture the vehicle dynamics reasonably good as it is depicted in Fig. 5 (dash line) in comparison with the experimental data (solid line). Furthermore, for validating the models all negative speeds have been ignored, as in practice they are physically impossible if one just combines first gear and the brake pedal action.
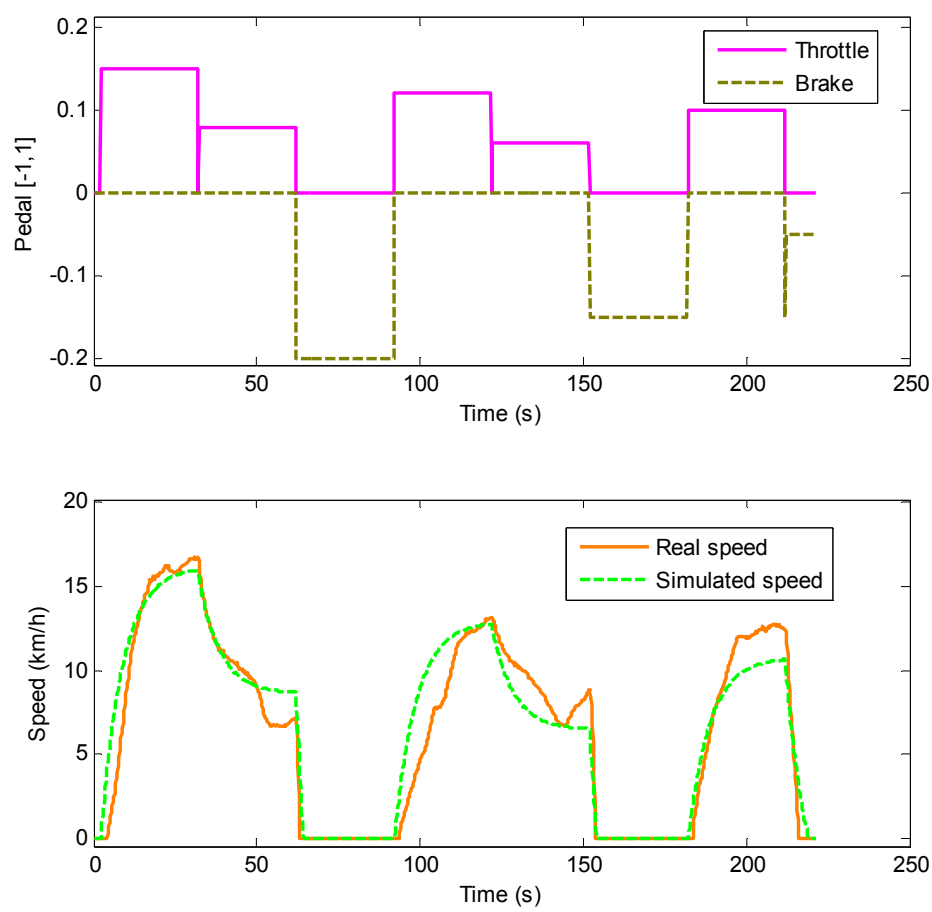

Fig. 5. Experimental car response and time-domain model validation.

\section{Design of the hybrid controller for the experimental vehicle}

This section will describe the application of hybrid GPC control strategy used for the car's speed control and the tuning of controllers. The goal of this strategy is to follow as precisely as possible a velocity profile used as reference by means of throttle and brake actions. Moreover, the control strategy must obey at any time the imposed constraints due to design decisions, security/comfort of the vehicle's occupants or mechanical restrictions.

To do so, the transfer functions (35) and (36) constitute the starting point in the controller tuning procedure, thus the control horizons are selected to capture their loop dominant dynamics. We have chosen a time window of 2 seconds ahead defined by 
$N_{1}=1$ and $N_{2}=10$, because it is appropriated for a low speed traffic scene. An unnecessary increment of $N_{2}$ would lead to a system with an excessively slow response. It would mean, in practice, that the vehicle could not stop in time and probably crash. On the other hand, we have fixed the value of control horizon $N_{u}$ at 1 obtaining a reasonable good response. A larger value of $N_{u}$ produced tighter control actions which led to inconvenient responses for both dynamics.

In order to improve the system robustness against the models-processes mismatches and disturbance rejections we have used prefilters (37) for both dynamics.

$$
T\left(z^{-1}\right)=\left(1-\rho z^{-1}\right)^{N_{1}}
$$

where $\rho$ has been chosen to be close to the dominant poles of (35) and (36) as it is recommended in the guideline [28]. Thus, the prefilters have the following expression:

$$
T_{t}\left(z^{-1}\right)=T_{b}\left(z^{-1}\right)=\left(1-0.9 z^{-1}\right)
$$

Once the controller horizons and the prefilter are chosen, the following step is finding the weighting sequences $(\gamma, \lambda)$. In this case, the pair $\left(1,10^{-6}\right)$ has been taken because we have obtained a reasonably good behaviour with it. Output response was barely affected by variations in parameter $\gamma$ and larger values of $\lambda$ means small changes in control signal that led the system to instability in the worst case scenario.

Finally, system constraints must be defined taking into account inherent vehicle issues, comfort and security criteria, and so on. Therefore, the sets of constraints for both dynamics: throttle and brake are presented below.

- Throttle constraints:

- Velocity constraint: low-speed vehicle responses are studied in this paper. For this reason, first gear is used during all the experiment. For engine protection purposes, maximum speed was limited to $(v \leq 20 \mathrm{~km} / \mathrm{h})$. Furthermore, all negative speeds are also not allowed. $(v \geq 0 \mathrm{~km} / \mathrm{h})$.

$$
\left[\frac{-G_{t}}{G_{t}}\right] \cdot \overline{\Delta u}(k) \leq\left[\frac{-\left(0-y_{F_{t}}\right)}{\left(20-y_{F_{t}}\right)}\right]
$$


- Acceleration constraint: The car response has to be smooth enough to guarantee that its acceleration remains always within interval $[-2,2] \mathrm{m} / \mathrm{s}^{2}$. It is the maximum acceptable acceleration for standing passengers in accordance with [36]. Considering the sampling time of the system is set to $5 \mathrm{~Hz}$, the maximum permitted increment of velocity is $\pm 1.44 \mathrm{~km} / \mathrm{h}$.

$$
\left[\frac{-\Delta G_{t}}{\Delta G_{t}}\right] \cdot \overline{\Delta u}(k) \leq\left[\frac{-\left(-1.44-\Delta y_{F_{t}}\right)}{1.44-\Delta y_{F_{t}}}\right]
$$

- Input constraints: the values of manipulated variable, Pedal, are normalized within the range of $[-1,1]$. So that,

$$
\left[\begin{array}{c}
-1 \\
1
\end{array}\right] \cdot \overline{\Delta u}(k) \leq\left[\begin{array}{c}
-(-1)+u(k-1) \\
1-u(k-1)
\end{array}\right]
$$

- Brake constraints:

- Velocity constraint: Negative speeds are not allowed. $(v>0 \mathrm{~km} / \mathrm{h})$. Thus,

$$
\left[-G_{b}\right] \cdot \overline{\Delta u}(k) \leq\left[-\left(0-y_{F_{b}}\right)\right]
$$

- Acceleration constraint: The maximum permitted increment of velocity is $\pm 1.44 \mathrm{~km} / \mathrm{h}$ as we have seen previously.

$$
\left[\frac{-\Delta G_{b}}{\Delta G_{b}}\right] \cdot \overline{\Delta u}(k) \leq\left[\frac{-\left(-1.44-\Delta y_{F_{b}}\right)}{1.44-\Delta y_{F_{b}}}\right]
$$

- Input constraints: In order to avoid abrupt braking operations, we have limited the maximum value of brake action to -0.15 . So that,

$$
\left[\begin{array}{c}
-1 \\
1
\end{array}\right] \cdot \overline{\Delta u}(k) \leq\left[\begin{array}{c}
-(-0.15)+u(k-1) \\
1-u(k-1)
\end{array}\right]
$$




\section{Experimental results}

Once the controller parameters have been tuned and checked, the following step is to test the behaviour of the proposed control strategy in a real environment with our production gasoline-propelled car (Citroën C3 Pluriel described previously).

The experimental trials was accomplished at the Centre for Automation and Robotics (CAR) that is a joint research centre of the Spanish Consejo Superior de Investigaciones Científicas and the Universidad Politécnica de Madrid. Its private driving circuit has been designed with scientific purposes and represents an inner-city area with bends, straight-road segments, and so on. Fig. 6 shows an aerial sight.

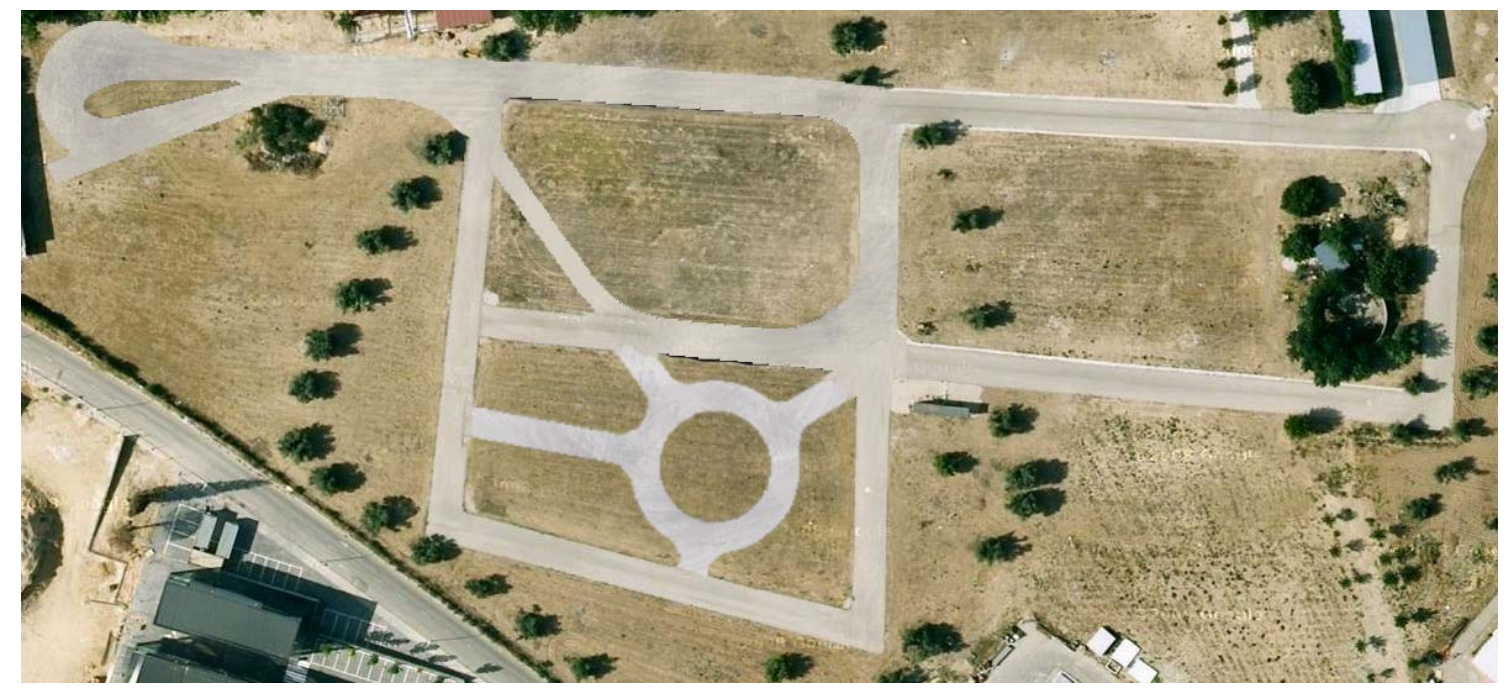

Fig. 6. Private driving circuit at CAR.

In order to validate our hybrid control strategy, three itineraries with different starting points within driving circuit were considered. Various target speed changes were also set by the on-board industrial PC at 25 or 30 seconds intervals while observing the low speed range; furthermore, the automatic gearbox was always maintained in the first gear, avoiding any effect of gear changes and forcing the car to move at low speed. As in previous works $[6,8,9]$, the speed error was kept close to zero.

The itinerary 1 is the longest one, where the vehicle runs following a route with several gentle slope changes, carrying out speed changes each 30 seconds.

The itinerary 2 is shorter than itinerary 1 , but it runs through the steep slope. Reference speed is changed each 25 seconds. 
The itinerary 3 is as long as itinerary 2. The vehicle runs following the flattest circuit part. The speed changes each 25 seconds and the changes have larger amplitudes.

Fig. 7 depicts the responses of the vehicle, both experimental --real time-- (green line) and simulated (blue line) in itinerary 1. The upper plot shows the vehicle's speed $(\mathrm{km} / \mathrm{h})$. The middle plot shows the action on the pedals (accelerator and brake), with the values in the range $[-1,0)$ to indicate an action on the brake and $[0,1]$ to indicate an action on the accelerator. Finally, the lower plot shows the comfort of standing passengers which is given by the acceleration $\left(\mathrm{m} / \mathrm{s}^{2}\right)$. Fig. 8 and Fig. 9 present the responses of the vehicle in itinerary 2 and 3, respectively. Our hybrid controller accomplished all practical requirements and constraints with a vehicle response stable, smooth and reasonably good in comparison with its simulation in all cases.

With respect to the comfort of the vehicle's occupants, it is observable that vehicle acceleration always remains within the maximum acceptable acceleration requirement \pm $2 \mathrm{~m} / \mathrm{s}^{2}$, due to the acceleration constraint set previously. Therefore, the comfort driving requirements are guaranteed.

Moreover, the proposed hybrid controller leads to a good system response even at very low speed $(4 \mathrm{~km} / \mathrm{h} \approx 1 \mathrm{~m} / \mathrm{s})$, where the nonlinear dynamics of engines of gasoline-propelled cars are specially significant. It is reached with a smooth control action as it is depicted in Fig. 7. This is a crucial situation which makes the designed hybrid controller performs very well to keep the engine running and avoid the car stalled. 

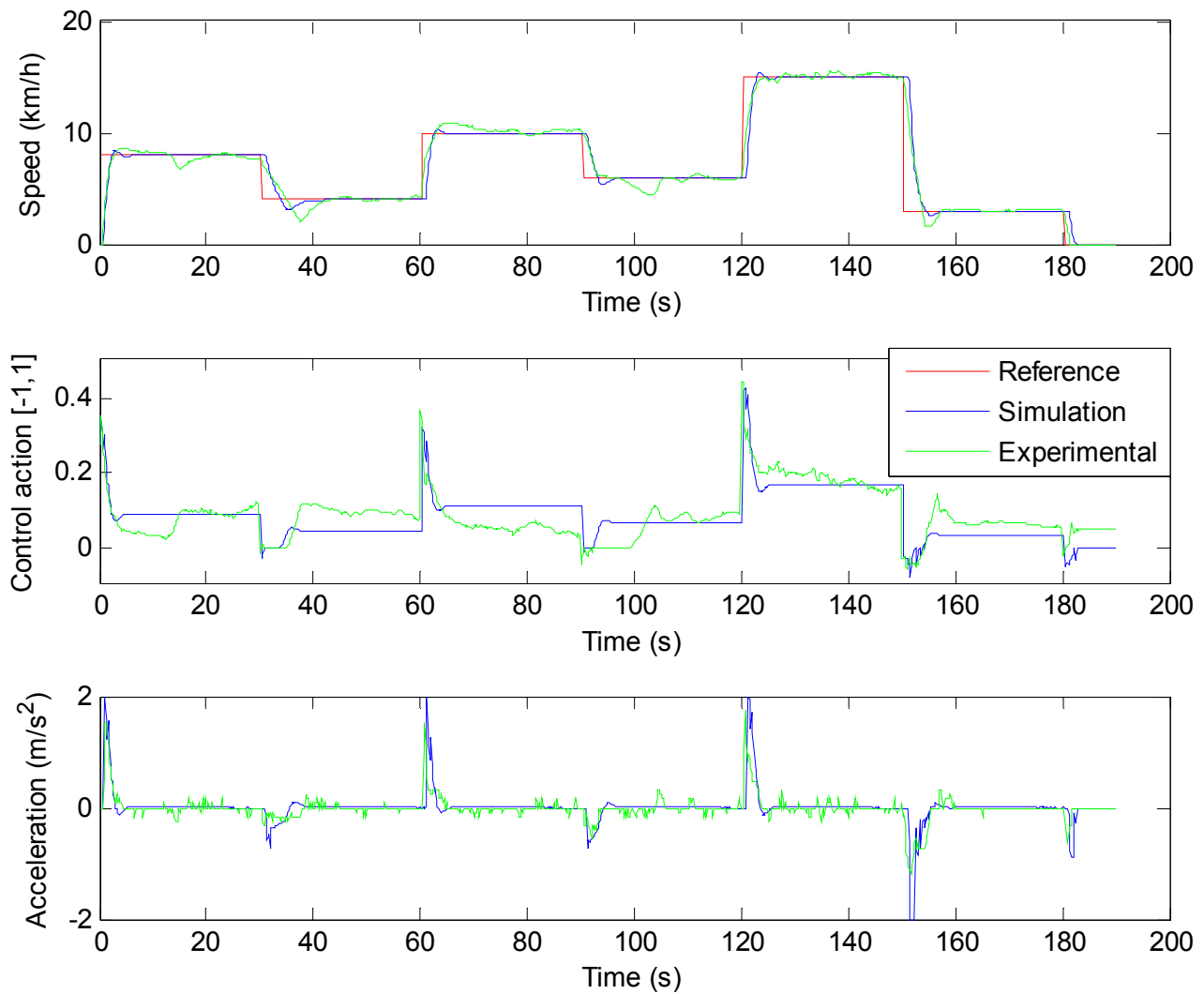

Fig. 7. Hybrid controller performance in itinerary 1. 

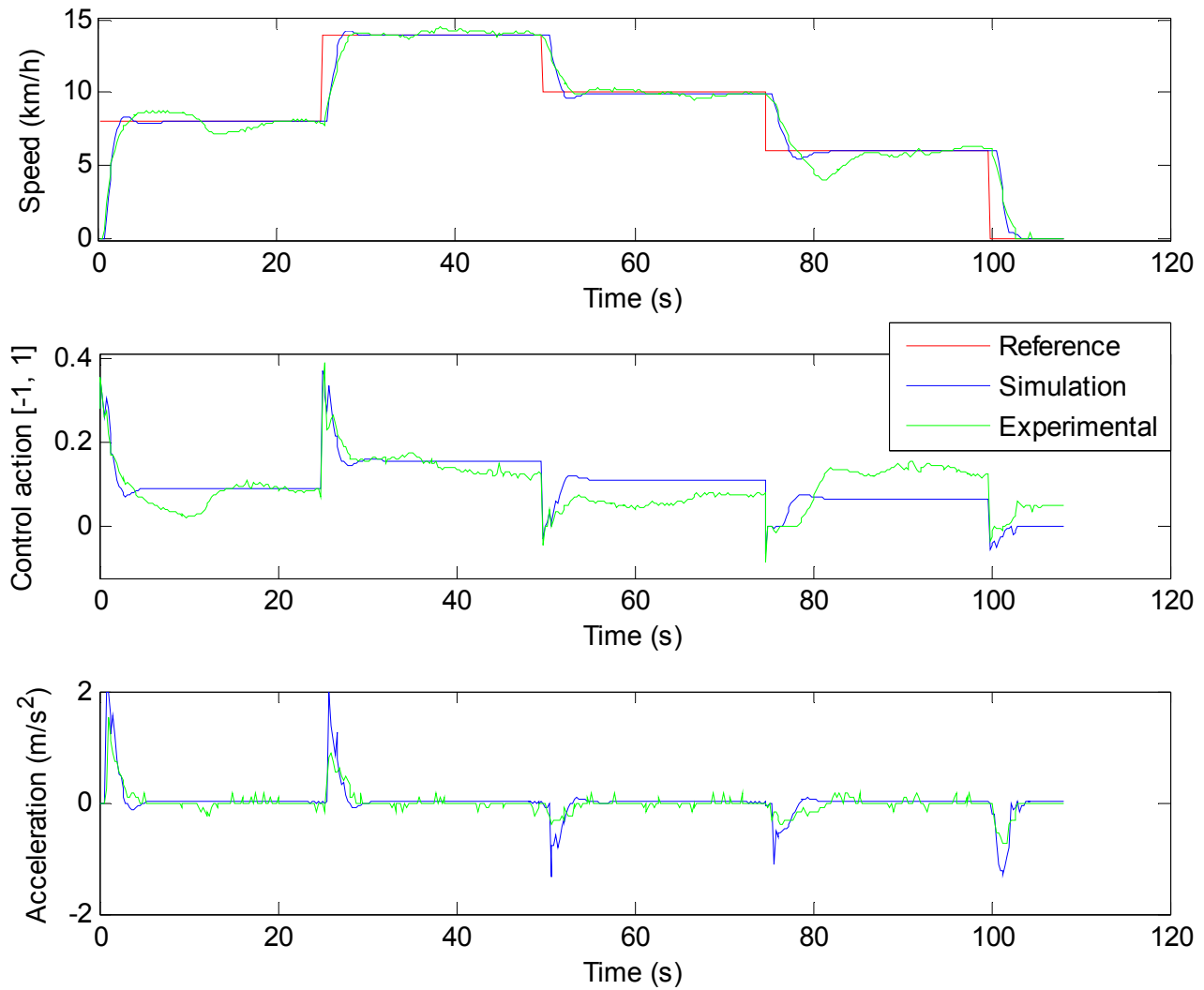

Fig. 8. Hybrid controller performance in itinerary 2. 

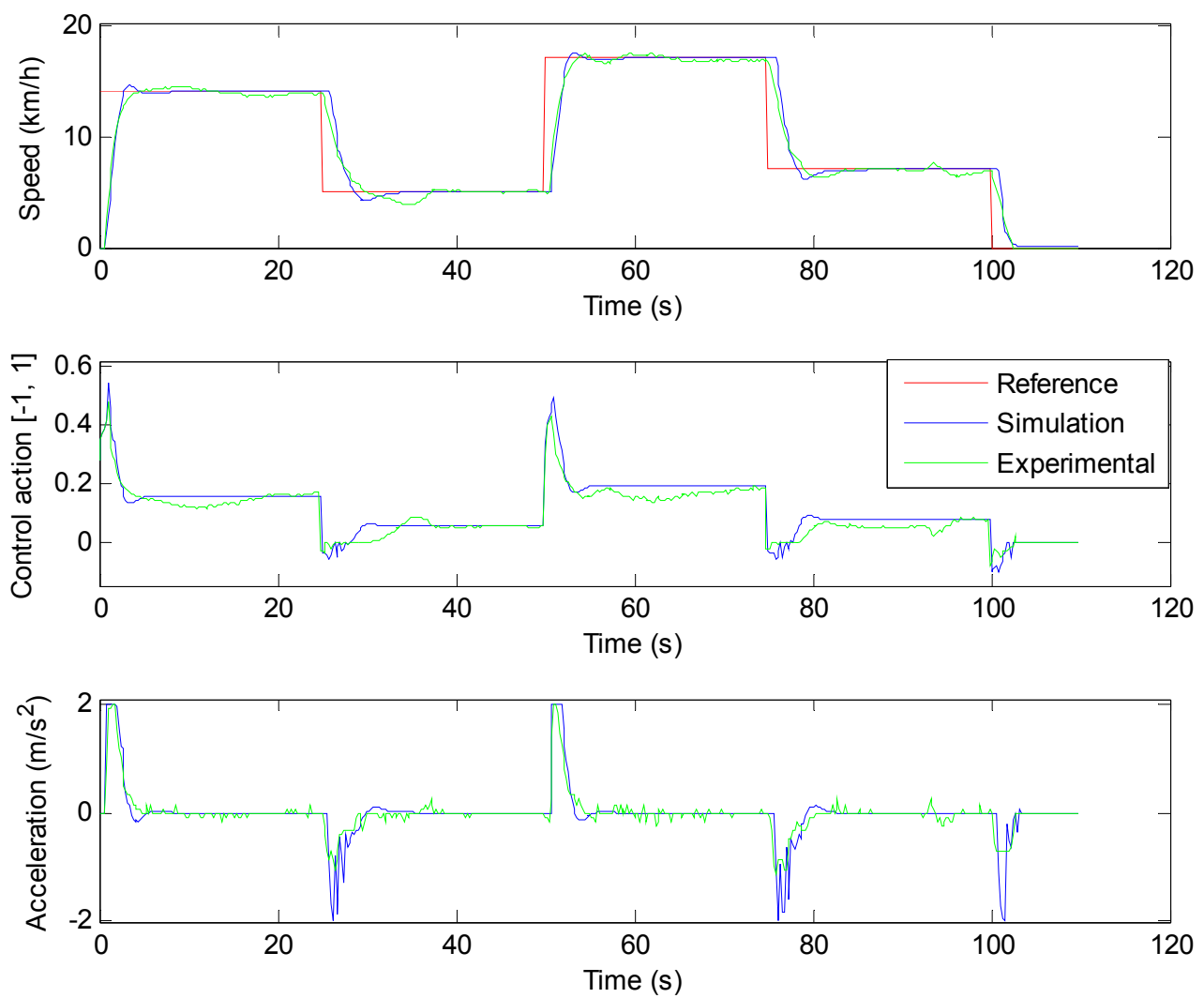

Fig. 9. Hybrid controller performance in itinerary 3.

In order to quantify these results, we will compare the principal control quality indicators of the hybrid control strategy proposed for each itinerary, that is, speed error (reference - experimental), smoothness of the control action, and acceleration. The last ones require to calculate the well-known Fast Fourier Transform (FFT) to estimate them.

The FFT (45) is an efficient algorithm to compute the Discrete Fourier Transform (DFT), $\mathfrak{F}$,

$$
U_{k}=\mathfrak{F}\left(u_{k}\right)=\sum_{i=0}^{N-1} u_{k} e^{\left(\frac{2 \pi N}{k_{i}}\right)}, \quad k=0, \ldots, N-1
$$

where $u_{k}$ is the control action or acceleration value at time $t_{k}$ and $N$ the length of these signals. FFT yields the signal sharpness by means of a frequency spectrum analysis of the sampled signal. 
A good indicator of the overall control action and acceleration signals with robustness to outliers is (46), where we have used the median $\tilde{u}$ of sequence $U_{k}$.

$$
P\left(U_{k} \leq \tilde{u}\right) \geq \frac{1}{2} \wedge P\left(U_{k} \geq \tilde{u}\right) \geq \frac{1}{2}
$$

We have also used the following widely statistics parameters to evaluate the speed error:

- mean:

$$
\bar{e}=\frac{1}{N} \sum_{k=0}^{N-1} e_{k}
$$

- standard deviation:

$$
\sigma=\sqrt{\frac{1}{N} \sum_{k=0}^{N-1}\left(e_{k}-\bar{e}\right)^{2}}
$$

- root mean square error:

$$
R M S E=\sqrt{\frac{1}{N} \sum_{k=0}^{N-1} e_{k}^{2}}
$$

where $e_{k}$ is the speed error at time $t_{k}$. Furthermore, we have also used the median $\tilde{e}$. These control quality indicators are reflected in Table I.

Table I: System performance for each itinerary.

\begin{tabular}{|c|c|c|c|c|c|c|}
\hline \hline \multirow{2}{*}{ Itinerary } & \multicolumn{4}{|c|}{ Speed error } & $\begin{array}{c}\text { Control } \\
\text { action }\end{array}$ & Acceleration \\
\cline { 2 - 7 } & Mean & St.dev. & Median & RMSE & FFT Median & FFT Median \\
\hline 1 & -0.013 & 1.3335 & -0.03 & 1.3329 & 0.3828 & 2.3206 \\
2 & 0.0351 & 1.3833 & 0.0 & 1.3825 & 0.1577 & 1.3597 \\
3 & 0.0852 & 2.4039 & 0.2 & 2.4032 & 0.2735 & 0.7783 \\
\hline
\end{tabular}

One observes that the speed error responses of vehicle in itinerary 1 and 2 are better than the response in itinerary 3 , due to the amplitude of speed changes are shorter in cases 1 and 2, so they need less time to reach the reference and steady state after speed changes (remember that it has been fixed a maximum increment of velocity). This is 
observable in Table I where, in terms of speed error, all statistic parameters of cases 1 and 2 are better than case 3 ones.

With respect to the control action, we obtain the worst results in itinerary 1, where the combination of amplitude of speed changes, curves and slopes changes have forced the hybrid controller to exhibit a control action that, in many cases, was larger than the one simulated.

Although the itinerary 3 presents larger amplitudes of speed changes, the flattest route has allowed the vehicle to get the best values in terms of the softness of the acceleration, with values very close to the ones obtained by means of simulation.

To sum up, simulations and experimental results showed that:

- The hybrid proposed controller behaves reasonably good when the experimental vehicle is following the speed reference, despite inevitable unmodeled dynamics and circuit perturbations.

- There are no big differences between simulations and experimental results in terms of speed error, control action and acceleration throughout the three proposed trials. Obviously, the more uncertainties and circuit perturbations exist, the poorer results are obtained.

- Standing passengers security and comfort is guaranteed at any time thanks to the imposed constraints. The system variables always remained below the maximum acceptable requirements.

\subsection{Comparison with other strategies}

This work presents a hybrid strategy based on a predictive control formulation which makes possible the use of process constraints. This fact is very important to impose the passengers security and comfort analytically in the uncomfortable scene of traffic jams. This valuable characteristic, the treatment of constraints, subjects the vehicle dynamics to compliance with design requirements (inputs and outputs constraints). Thus, it is not easy to propose a direct comparison between the hybrid predictive control scheme and other control strategies whose manipulated and controlled variables are not subjected to the same set of constraints. Moreover, there exist many control strategies that have 
demonstrated their performance with simple or complex plant models in simulation, but their formulations cannot be embedded in the car hardware in a straightforward way.

Nevertheless, in order to show the better performance of adopted solution versus other control strategies, we have proposed a comparison between our hybrid method and a fuzzy controller designed for this vehicle that deals with comfort requirements. Moreover, the results of a human driver who used the pedals to adapt the car speed to the different target speeds are also added. Both fuzzy controller and human results was published previously in [37].

The test scenario includes four speed changes of $10 \mathrm{~km} / \mathrm{h}, 15 \mathrm{~km} / \mathrm{h}, 20 \mathrm{~km} / \mathrm{h}$, and $25 \mathrm{~km} / \mathrm{h}$ for 60 seconds.

Table II shows the comparison results in terms of speed error (49) after the first five seconds (i.e., once the system reached the steady state) for the different speed requirements.

Table II. Comparative error table.

\begin{tabular}{|c|c|c|c|}
\hline \hline Speed Error & Hybrid controller & Fuzzy controller [37] & Human driver [37] \\
\hline at $10 \mathrm{~km} / \mathrm{h}$ & 0.43 & 0.71 & 0.63 \\
at $15 \mathrm{~km} / \mathrm{h}$ & 0.29 & 0.98 & 0.88 \\
at $20 \mathrm{~km} / \mathrm{h}$ & 0.38 & 0.84 & 0.72 \\
\hline at $25 \mathrm{~km} / \mathrm{h}$ & 0.47 & 0.90 & 1.22 \\
\hline
\end{tabular}

One can appreciate how the proposed hybrid controller improves the performance obtained by either the fuzzy controller or a human driver when it comes to keep a constant low-speed, demonstrating the good behaviour of the proposed system that keeps all errors below 0.5 .

\section{Conclusions}

This paper has focused on the use of an hybrid predictive control strategy based on a transfer function model to manage autonomously a real gasoline car at low speed conditions. Its highly nonlinear dynamics has supposed an excellent test bed for applying the beneficial characteristics of proposed hybrid controller. 
In order to satisfy such control objectives as driving at low speed range trying to keep the speed error close to zero, smoothness of the control action, comfortable acceleration for the car's passengers,...; both the controller parameters (weighting sequences, horizon parameters and polynomial filter $T$ ) and the set of constraints have been particularly tuned for the two predictive controllers that integrate this hybrid system. It has been done in accordance with the two dynamical behaviours involved: throttle and brake.

Finally, the proposed controller have been trialled following three itineraries with different starting points and various target speed changes, which recreate the common low speed situation in traffic jams. All control requirements have been successfully achieved in spite of inevitable uncertainties and circuit perturbations. Additionally, the controller has been compared with other strategies, showing a better performance in terms of speed error. Thus, we can consider that the proposed hybrid control strategy is valid, appropriate and recommendable to face the challenging control topic described in this work.

\section{Acknowledgements}

The authors wish to acknowledge the economical support of the Spanish Distance Education University (UNED), under project reference PROY29 (Proyectos Investigación 2012), and the AUTOPIA Program of the Center for Automation and Robotics UPM-CSIC.

\section{References}

[1] N.B. Hounsell, B. P. Shrestha, J. Piao, M. McDonald, Review of urban traffic management and the impacts of new vehicle technologies, IET Intelligent Transport Systems, vol. 3, no. 4, pp. 419-428, 2009.

[2] S. Moon, I. Moon, K. Yi, Design, tuning, and evaluation of a full-range adaptive cruise control system with collision avoidance, Control Engineering Practice, vol. 17, no. 4, pp. 442-455, 2009. 
[3] T. Aono, T. Kowatari, Throttle-control algorithm for improving engine response based on air-intake model and throttle-response model, IEEE Trans. on Industrial Electronics, vol. 53, no. 3, pp. 915-921, 2006.

[4] L. van Kampen, Cruise control in personenautos: een literatuurorientatie op verkeersveiligheidsaspecten (cruise control in passenger cars), SWOV, The Netherlands, Tech. Rep., 1996.

[5] J. Pérez, F. Seco, V. Milanés, A. Jiménez, J.C. Díaz, T. de Pedro, An RFID-based intelligent vehicle speed controller using active traffic signals, Sensor, vol. 10, pp. $5872-5887,2010$.

[6] V. Milanés, J. Villagrá, J. Pérez, C. González, Low-speed longitudinal controllers for mass-produced cars: a comparative study, IEEE Transactions on Industrial Electronics, vol. 59, no. 1, pp. 620-628, 2012.

[7] M. A. Sotelo, D. Fernández, J. E. Naranjo, C. González, R. García, T. de Pedro, J. Reviejo, Vision-based adaptive cruise control for intelligent road vehicles, in Proc. IEEE International Conference on Intelligent Robots and Systems, Sendai, Japan, pp. 64-69, 2004.

[8] I. Tejado, V. Milanés, J. Villagrá, J. Godoy, H. HosseinNia, B. M. Vinagre, Low speed control of an autonomous vehicle by using fractional PI, in Proc. 18th IFAC World Congress, Milano, Italy, pp. 15025-15030, 2011.

[9] M. Romero, A.P. de Madrid, C. Mañoso, V. Milanés, B.M. Vinagre, Fractionalorder generalized predictive control: application for low-speed control of gasolinepropelled cars, Mathematical Problems in Engineering, vol. 2013, Article ID 895640, 2013.

[10] J . M. Maciejowski, Predictive control with constraints, Prentice Hall, United Kingdom, 2002.

[11] J . A. Rossiter, Model based predictive control. A practical approach, CRC Press LLC, 2003. 
[12] E . F. Camacho, C. Bordóns, Model predictive control, $2^{\text {nd }}$ Edition. Springer, USA, 2004.

[13] J. Lygeros, An overview of hybrid systems control, Handbook of networked and embedded control systems, pp. 519-537, D. Hristu-Varsakelis and W.S. Levine (Eds.), Birkhäuser, Boston, USA, 2005.

[14] R.L. Grossmann, A. Nerode, A.P. Ravn, H. Rischel, Hybrid Systems, Lecture notes in computer science, vol. 736 , Springer Verlag, New York, USA, 1993.

[15] R. Alur, C. Courcoubetis, N. Halbwachs, T.A. Hezinger, P.H. Ho, X. Nicollin, A. Olivero, J. Sifakis, S. Yovine, The algorithmic analysis of hybrid systems, Theoretical Computer Science, vol. 138, no.1, pp. 3-34, 1995.

[16] J. Lygeros, Lecture notes on hybrid systems, D. electrical and computer engineering (University of Patras), Patras, Greece, 2004.

[17] A. Bemporad, M. Morari, Control of systems integrating logic, dynamics, and constraints, Automatica, vol. 35, no. 3, pp. 407-427, 1999.

[18] B. Potočnik, G. Mušič, B. Zupančič, Model predictive control of discrete-time hybrid systems with discrete inputs, ISA Transactions, vol. 44, no. 2, pp. 199-211, 2005.

[19] B. Potočnik, G. Mušič, I. Škrjanc, B. Zupančič, Model predictive control of hybrid systems: A probabilistic neural-network approach to real-time control, Journal of Intelligent and Robotic Systems, vol. 51, no. 1, pp. 45-63, 2008.

[20] M. Kvasnica, Real-time model predictive control via multi-parametric programming. Theory and Tools, VDM Verlag Publishing, 2009.

[21] M. Kvasnica, P. Grieder, M. Baotić, F.J. Christophersen, Multi-parametric Toolbox, http://control.ee.ethz.ch/ mpt/, 2006.

[22] A. Bemporad, Hybrid toolbox for real-time applications user's guide, bemporad@ing.unitn.it, 2010. 
[23] T. Geyer, G. Papafotiou, M. Morari, Model predictive control in power electronics: A hybrid systems approach, in Proc. 44th IEEE Conference on Decision and Control, Seville, Spain, pp. 5606-5611, 2005.

[24] N.H. El-Farra, P. Mhaskar, P.D. Christofides, Hybrid predictive control of nonlinear systems: Method and applications to chemical processes, International Journal of Robust and Nonlinear Control, vol. 14, pp. 199-225, 2004.

[25] D. Sáez, C.E. Cortés, A. Núñez, Hybrid adaptive predictive control for the multivehicle dynamic pick-up and delivery problem based on genetic algorithms and fuzzy clustering, Computers \& Operations Research, vol. 35, no. 11, pp. 3412-3438, 2008.

[26] D. W. Clarke, C. Mohtadi, P.S. Tuffs, Generalized predictive control. Part I. The basic algorithm, Automatica, vol. 23, no. 2, pp. 137-148, 1987.

[27] D .W. Clarke, C. Mohtadi, P.S. Tuffs, Generalized predictive control. Part II. Extensions and interpretations, Automatica, vol. 23, no.2, pp. 149-160, 1987.

[28] T.W. Yoon, D.W. Clarke, Observer design in receding-horizont control, International Journal of Control, vol. 61, no. 1, pp. 171-191, 1995.

[29] D .W. Clarke, C. Mohtadi, Properties of generalized predictive control, Automatica, vol. 25, pp. 859-875, 1989.

[30] S. Huand, W. Ren, Vehicle longitudinal control using throttles and brakes, Robotics and Autonomous Systems, vol. 26, no. 4, pp. 241-253, 1999.

[31] P. Shakouri, A. Ordys, M.R. Askari, Adaptive cruise control with stop\&go function using the state-dependent nonlinear model predictive control approach, ISA Transactions, vol. 51, no. 5, pp. 622-631, 2012.

[32] M. Romero, I. Tejado, A.P. de Madrid, B.M. Vinagre, Tunning predictive controllers with optimization: Application to GPC and FGPC, in Proc. 18th IFAC World Congress, Milano, Italy, pp. 10824-10829, 2011. 
[33] V. Milanés, C. González, J.E. Naranjo, E. Onieva, T. de Pedro, Electro-hydraulic braking system for autonomous vehicles, International Journal of Automotive Technology, vol. 11, no. 1, pp. 89-95, 2010.

[34] V. Milanés, D.F. Llorca, B.M. Vinagre, C. González, M.A. Sotelo, Clavileno: evolution of an autonomous car, in Proc. 13th IEEE International Intelligent Transportation Systems, Madeira, Portugal, pp. 1129-1134, 2010.

[35] Mathworks Inc., Matlab identification toolbox user's guide, 2007.

[36] BECHTEL, Compendium of executive summaries from the maglev system concept definition final reports, Tech. Rep., U.S. Department of Transportation, http://ntl.bts.gov/DOCS/CES.html, 1993.

[37] E. Onieva, V. Milanés, C. González, T. de Pedro, J. Pérez and J. Alonso, Throttle and brake pedals automation for populated areas, Robotica, vol. 28, no. 4, pp. 509-516, 2010. 